\title{
Modeling persistent temporal patterns in dissociated cortical cultures using reservoir computing
}

Tayfun Gürel ${ }^{1,2,3^{*}}$, Samora Okujeni ${ }^{1,2,4}$, Oliver Weihberger ${ }^{1,2,4}$, Stefan Rotter ${ }^{1,5}$, Ulrich Egert ${ }^{1,2}$

From Nineteenth Annual Computational Neuroscience Meeting: CNS*2010

San Antonio, TX, USA. 24-30 July 2010

Persistent spatiotemporal patterns have been observed extensively in various neural systems including cortical cultures [1]. Activity in cortical cultures is composed of network-wide bursts of spikes, during which global firing rate increases dramatically. Previously, it has been shown that cultures display persistent temporal patterns that are hierarchically organized and stable over several hours. Fluctuations in the culture activity persistently converge to stable precise temporal patterns, for which these patterns are called dynamic attractors. Temporal structure in network bursts can be clustered into several groups, each of which can be seen as a separate burst type.

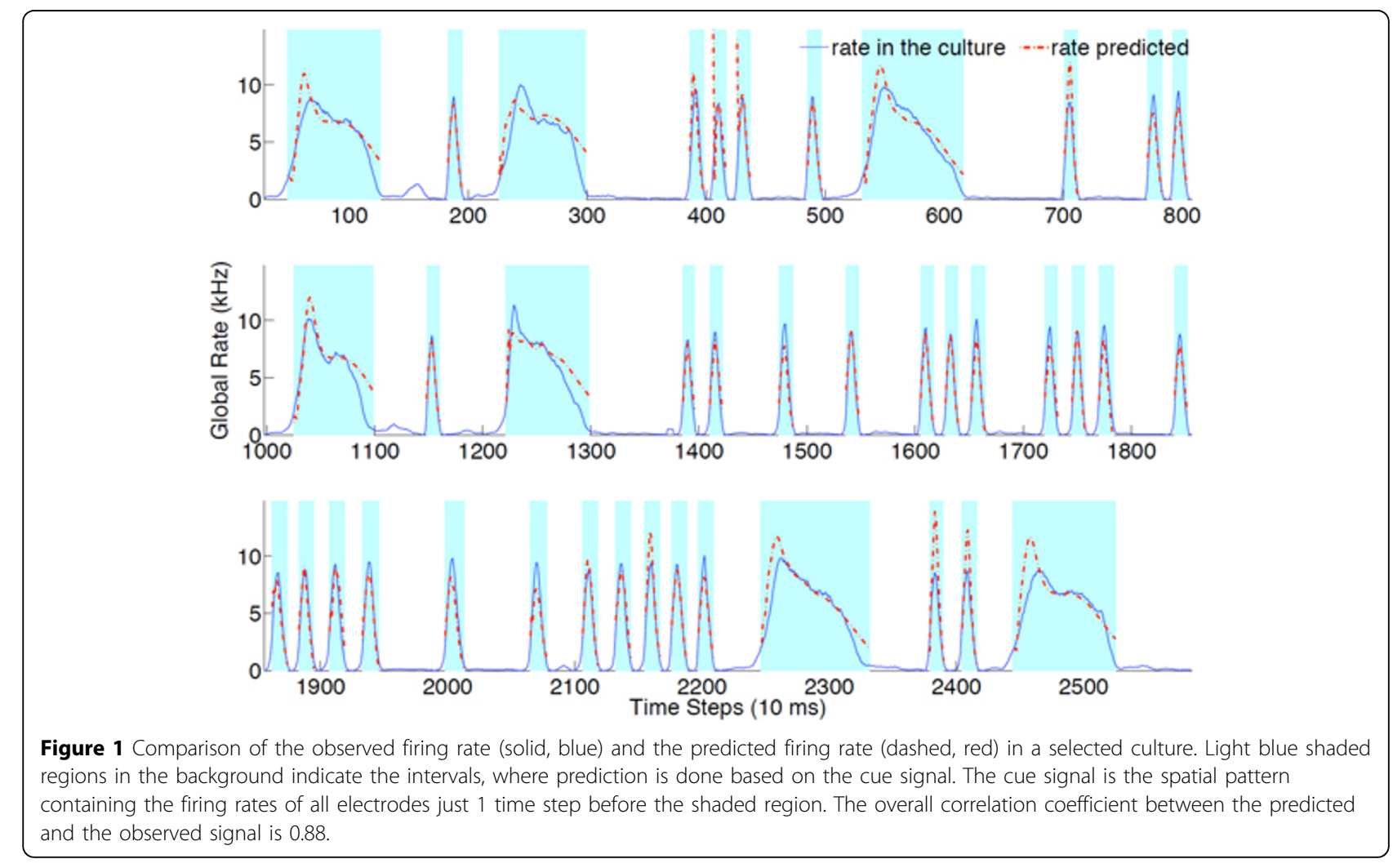

\footnotetext{
* Correspondence: guerel@informatik.uni-freiburg.de

1 Bernstein Center Freiburg, Albert-Ludwig University of Freiburg, Germany
} 
A model of a neural system should be able to reproduce the temporal patterns under the same input and/or initial state, which is a minimal requirement for a network-level model to reveal the information encoded in such patterns. Our approach taken here is to employ a generic model (a reservoir network) that displays a rich repertoire of complex spatiotemporal patterns to be matched with the observed biological patterns by parameter tuning. More specifically, we employ an Echo State Network (ESN) [2] with leaky integrator neurons as a modeling tool. Here, we consider cultures of dissociated cortical tissue recorded with microelectrode arrays (MEA) as an example of biological neural networks without specific connectivity and simulate the corresponding burst types based on a cue signal. The cue signal is composed of a snapshot $(10 \mathrm{~ms})$ of the individual firing rates recorded at each electrode at burst onset and serves as an indicator of the current dynamic state of the network. A simple readout training of the ESN yields a predictive model of the temporal activity pattern in the global firing rate. The simulated pattern displays a high correlation with the actual one observed in the culture (Figure 1). The model can also be used to visualize the underlying structure in the recorded signals.

\section{Acknowledgements}

This work was supported by the German BMBF (BCCN Freiburg, 01GQ0420).

\section{Author details}

${ }^{1}$ Bernstein Center Freiburg, Albert-Ludwig University of Freiburg, Germany. ${ }^{2}$ Dept. of Microsystems Engineering - IMTEK, Albert-Ludwig University of Freiburg, Germany. ${ }^{3}$ Faculty of Biology, Albert-Ludwig University of Freiburg, Germany. ${ }^{4}$ Neurobiology and Biophysics, Faculty of Biology, Albert-Ludwig University of Freiburg, Germany. ${ }^{5}$ Computational Neuroscience, Faculty of Biology, Albert-Ludwig University of Freiburg, Germany.

Published: 20 July 2010

\section{References}

1. Wagenaar DA, Nadasdy Z, Potter SM: Persistent dynamic attractors in activity patterns of cultured neuronal networks. Phys Rev E Stat Nonlin Soft Matter Phys 2006, 73(5 Pt 1):051907.

2. Jaeger $\mathrm{H}$ : The "echo state" approach to analysing and training recurrent neural networks. GMD Report 148, GMD - German National Research Institute for Computer Science 2001

doi:10.1186/1471-2202-11-S1-P42

Cite this article as: Gürel et al:: Modeling persistent temporal patterns in dissociated cortical cultures using reservoir computing. BMC

Neuroscience 2010 11(Suppl 1):P42.

\section{Submit your next manuscript to BioMed Central} and take full advantage of:

- Convenient online submission

- Thorough peer review

- No space constraints or color figure charges

- Immediate publication on acceptance

- Inclusion in PubMed, CAS, Scopus and Google Scholar

- Research which is freely available for redistribution

Submit your manuscript at www.biomedcentral.com/submit 